\title{
Robotic single-port surgery
}

\author{
Hye-Sung Moon
}

Department of Obstetrics and Gynecology, College of Medicine, Ewha Womans University, Seoul, Korea

In the gynecological field, minimally invasive surgery is becoming increasingly popular due to its short hospitalization period, rapid recovery, and cosmetic benefits $[1,2]$. With the U.S. Food and Drug Administration approval of the da Vinci® Surgical System (Intuitive Surgical Inc., Synnyvale, CA, USA), robotic surgery (RS) has become widespread to overcome the shortcomings of laparoscopic surgery, such as collisions and clashing of instruments and limited range of motion [3]. Introduction of RS has numerous benefits, including less postoperative pain, the reduced blood loss during surgery, and shorter hospital stay.

In 2013, a single-site platform of da Vinci Si ${ }^{\circledR}$ Surgical System was introduced for performance of gynecologic procedures through a single umbilical incision similar to laparoendoscopic single-site surgery. Robotic single-site surgery (RSSS) maintains the advantages of the RS that were already mentioned above, but in addition, the patient's cosmetic satisfaction for a single site scar on the umbilicus remains high and the complications related to accessory port sites can be avoided [4]. Although the feasibility and safety of RSS surgery using the da Vinci ${ }^{\circledR}$ Si or Xi Surgical System have been investigated in hysterectomy or myomectomy, there are still limitations due to semi-rigid and non-articulating instruments with lack of triangulation in those systems [5-7]. In order to per- form more robotic single-site surgeries successfully, we must develop good surgical skills and better suturing techniques with the current set of limited instruments [7-9].

Therefore, the da Vinci ${ }^{\circledR}$ SP Surgical System was recently introduced in 2018 to overcome the limitations of RSSS using the da Vinci ${ }^{\circledR}$ Si or Xi Surgical System. The new da Vinci ${ }^{\circledR}$ SP System enabled sufficient articulation with fully wristed, double-jointed instruments, and included a flexible three-dimensional camera. The surgeons were able to perform meticulous sutures comfortably with all three instruments of the da Vinci ${ }^{\circledR}$ SP Surgical System even through a single umbilical incision [10]. Preliminary data were reported that robotic singleport surgery (RSPS) using the da Vinci ${ }^{\circledR}$ SP Surgical System might be an alternative surgical technique for various benign gynecologic diseases. Especially, robotic single-port myomectomy (RSPM) using the da Vinci SP ${ }^{\circledR}$ Surgical System is a feasible surgical modality for women with symptomatic myoma and is expected to increase indications of single port myomectomy with following advantages (in submission).

Single-port instruments have sufficient mechanical power to hold and suture thicker tissues than previous Single-site instruments. The utilization of three instruments separately from the camera is possible. Therefore,

- Received:September 20, 2021 • Accepted: September 21, 2021

- Correspondening author: Hye-Sung Moon

Department of Obstetrics and Gynecology, College of Medicine, Ewha Womans University, 260 Gonghang-daero, Gangseo-gu, Seoul 07804, Korea E-mail:hsmoon00110@gmail.com

This is an Open Access article distributed under the terms of the Creative Commons Attribution Non-Commercial License (http://creativecommons.org/ licenses/by-nc/4.0) which permits unrestricted non-commercial use, distribution, and reproduction in any medium, provided the original work is properly cited. 
surgeon can handle the target more easily than before. In addition, it is relatively easy for the assistant to use additional laparoscopic instruments. Surgical motion in almost any direction is possible with all the single-port instruments capable of articulation over 90 degrees by two joints, in addition to an articulating camera. But, there are still some improvements to be made; for robotic instruments to function properly; two joints of instruments must be folded, and it is essential that instruments are introduced with a sufficient distance to the target. Therefore, RSPS may be ergonomically difficult to be done for huge sized targets.

For single-incision robotic sacrocolpopexy, surgical outcomes such as operative time and suturing time using da Vinci SP Surgical System were better than those using da Vinci Si or Xi Surgical System [11]. For single-incision robotic myomectomy, surgical outcomes such as operative time and estimated blood loss using da Vinci SP Surgical System were better than previous systems due to reduction of docking time, and more delicate surgery. The increased probability of getting better outcome may also be caused by the shortening of learning curve (in submission).

In comparison of multi-site robotic surgeries, surgical outcomes of robotic single-port hysterectomy or RSPM were not statistically different in myoma cases with the largest diameter less than $6 \mathrm{~cm}$ and the mean weight $<300 \mathrm{~g}$. It means that RSPM does not provide surgeon with disadvantages in comparison to the multiport approach in the carefully selected cases. Type of robotic approach is not an important factor for perioperative complication if well-developed robotic surgical system is available with more skilled operative techniques (in submission).

RSPS is a valuable and safe surgical type in gynecologic disease and da Vinci SP Surgical System proves to be a useful and viable system that expanded indications of robotic single-site platforms while overcoming limitations of da Vinci Xi and da Vinci Si systems. Further prospective clinical trials are still warranted to confirm the clinical guidelines. In the future, we are expecting that the surgical environments would develop further by emerging data, and imaging integration, and improvement of surgical system with additional reduction of cost, we can expect to be satisfied with better surgical outcomes.

\section{References}

1. Advincula AP, Wang K. Evolving role and current state of robotics in minimally invasive gynecologic surgery. J Minim Invasive Gynecol 2009;16:291-301.

2. Liu H, Lu D, Wang L, Shi G, Song H, Clarke J. Robotic surgery for benign gynaecological disease. Cochrane Database Syst Rev 2012;15:CD008978.

3. Gala RB, Margulies R, Steinberg A, Murphy M, Lukban J, Jeppson P, et al. Systematic review of robotic surgery in gynecology: robotic techniques compared with laparoscopy and laparotomy. J Minim Invasive Gynecol 2014;21:353-61.

4. Bogliolo S, Ferrero S, Cassani C, Musacchi V, Zanellini F, Dominoni $M$, et al. Single-site versus multiport robotic hysterectomy in benign gynecologic diseases: a retrospective evaluation of surgical outcomes and cost analysis. J Minim Invasive Gynecol 2016;23:603-9.

5. Buckley de Meritens A, Kim J, Dinkelspiel H, Chapman-Davis E, Caputo T, Holcomb KM. Feasibility and learning curve of robotic laparoendoscopic single-site surgery in gynecology. J Minim Invasive Gynecol 2017;24:323-8.

6. Matthews CA. New developments in robotics and single-site gynecologic surgery. Clin Obstet Gynecol 2017;60:296-311.

7. Choi EJ, Rho AM, Lee SR, Jeong K, Moon HS. Robotic single-site myomectomy: clinical analysis of 61 consecutive cases. J Minim Invasive Gynecol 2017;24:632-9.

8. Moon HS. Tips on robotic single-site surgery suture technique: screwing and clockwise direction suture technique for robotic single-site surgery. Taiwan J Obstet Gynecol 2018;57:432-4.

9. Yoo HK, Cho A, Cho EH, Kim SJ, Shim JE, Lee SR, et al. Robotic single-site surgery in benign gynecologic diseases: experiences and learning curve based on 626 robotic cases at a single institute. J Obstet Gynaecol Res 2020;46:1885-92.

10. Shin HJ, Yoo HK, Lee JH, Lee SR, Jeong K, Moon HS. Robotic

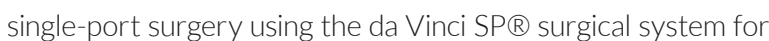
benign gynecologic disease: a preliminary report. Taiwan J Obstet Gynecol 2020;59:243-7.

11. Lee SR, Roh AM, Jeong K, Kim SH, Chae HD, Moon HS. First report comparing the two types of single-incision robotic sacrocolpopexy: single site using the da Vinci Xi or Si system and single port using the da Vinci SP system. Taiwan J Obstet Gynecol 2021;60:60-5. 